\title{
Criminologie
}

\section{L’aide juridique, dix ans après}

\section{Laurent Laplante}

Volume 15, numéro 1, 1982

Droit et justice

URI : https://id.erudit.org/iderudit/017147ar

DOI : https://doi.org/10.7202/017147ar

Aller au sommaire du numéro

Éditeur(s)

Les Presses de l'Université de Montréal

ISSN

0316-0041 (imprimé)

1492-1367 (numérique)

Découvrir la revue

Citer cet article

Laplante, L. (1982). L'aide juridique, dix ans après. Criminologie, 15(1), 9-20. https://doi.org/10.7202/017147ar d'utilisation que vous pouvez consulter en ligne.

https://apropos.erudit.org/fr/usagers/politique-dutilisation/ 
Presque une décennie après sa création, l'aide juridique québécoise se retrouve, en substance, au même carrefour qu'à sa naissance. Plus forte, assurément, mais battue par les mêmes vents. Elle soulève, en effet, les mêmes oppositions de la part des mêmes personnes et des mêmes intérêts. Elle satisfait à peu de choses près les espoirs qu'elle avait suscités. Elle justifie en revanche plusieurs des craintes qu'on pouvait entretenir à son endroit. Elle n'a, en somme, ni raté son coup, ce qui est heureux, ni substantiellement réformé la société, ce qui était sans doute immensément prévisible. Assurée désormais de la survie et même promise à l'irrésistible gonflement des grandes organisations, il lui reste à décider si elle éprouve le besoin d'un deuxième souffle et à quelles conditions elle laisserait ce souffle l'emporter.

\section{L'ACCES A LA MATURITE}

L'aide juridique québécoise est devenue une grosse affaire. Le réseau coûte une quarantaine de millions, occupe plus de 800 personnes, dont 325 avocats permanents, et confie des mandats à un nombre imposant d'avocats et de notaires de pratique privée.

La clientèle aussi a pris un gabarit industriel. Depuis quelque temps, on dépasse assez allègrement le seuil des 200000 dossiers par an et c'est donc en termes de millions qu'on dénombrera les contacts entre le public et le personnel de l'aide juridique.

La diversité des interventions saute, elle aussi, aux yeux. L'aide juridique intervient désormais aussi bien dans le secteur matrimonial que dans les causes criminelles, aussi bien dans les recours collectifs que devant les tribunaux administratifs. Certains secteurs, certes, reçoivent moins souvent la visite des mandataires de l'aide juridique, mais rien d'autre que l'insuffisance des ressources ne motive une telle orientation.

Pour en arriver à cette performance, il aura évidemment fallu que l'aide juridique québécoise sache d'abord se faire connaître et parvienne ensuite à livrer la marchandise. Sur les deux fronts, on peut parler de succès :

- L'information dispensée par l'aide juridique québécoise

\footnotetext{
* Directeur de la Régie des services publics du Québec.
} 
frappe, en effet, par son dynamisme, son originalité, sa grande liberté. Depuis la bande dessinée jusqu'au commercial télévisé, depuis la série d'affaires publiques jusqu'au photoroman, toutes les techniques sont mises à contribution et toutes empruntent le style et le ton qui peuvent rejoindre le mieux le public visé.

- Une fois le besoin créé dans le public, il s'agissait de ne décevoir personne. Le défi, en substance, a été relevé. Il faut, en effet, considérer comme important et significatif l'indice de satisfaction obtenu dans les sondages auprès des bénéficiaires. Que l'on soit content des services obtenus et que ce contentement atteigne des pourcentages de l'ordre de $80 \%$, tout cela témoigne éloquemment en faveur de l'aide juridique.

D'autres indices, à vrai dire, sont peut-être plus probants encore. Citons, entre autres, la réaction policière : elle est faite de déception et de colère devant l'exaspérante détermination des procureurs de l'aide juridique et devant l'impuissance des procureurs de la Couronne à tenir tête aux offensives de l'aide juridique. Citons également la réaction du Barreau : loin de contester de façon frontale le régime public d'aide juridique, il se borne à souhaiter qu'un plus fort pourcentage des dossiers soit acheminé vers la pratique privée et que soit relevé le tarif applicable aux mandats que reçoivent les praticiens du secteur privé.

Tout cela projette l'image d'un service public rapidement parvenu à la taille adulte et à la performance professionnelle.

\section{TROIS GRIEFS D'INEGALE PERTINENCE}

Cette maturation de l'aide juridique ne s'effectue assurément pas sans heurts, ni sans oppositions. La critique a même réussi, avec le temps, à mettre au point une variété de reproches dont plusieurs méritent autre chose qu'un haussement d'épaules. Les plus courants sont la rapide croissance des coûts, la bureaucratisation de la machine et surtout la passivité que maintient et accroît chez les bénéficiaires le recours à l'aide juridique.

Les deux premiers griefs ne résistent guère à l'examen. Le troisième, en revanche, soulève des questions encore mal bridées.

\section{Les coûts}

Que les coûts de l'aide juridique croissent rapidement, la démonstration en est faite. La dizaine de millions que l'on estimait suffisante au départ ne comblerait aujourd'hui qu'un quart des frais. Cette hausse et le fait que la mode vilipende volontiers les ser- 
vices publics qu'elle soupçonne de dépenser sans compter, expliquent sans doute pourquoi le gouvernement du Québec a tenu à scruter de près cette évolution des coûts. D'autant plus que les ténors du libéralisme tout azimut laissent toujours entendre qu'il en coûterait moins cher de s'en remettre à l'initiative privée plutôt qu'à des avocats fonctionnarisés.

C'est donc dans un contexte de comparaison qu'on a voulu situer la performance de l'aide juridique non plus seulement sur le plan du succès judiciaire ou social, mais encore sur le terrain du rendement économique. En demandant le coût d'un dossier d'aide juridique, on voulait en faire savoir si l'avocat permanent peut traiter ce dossier à moins cher que l'avocat de pratique privée.

Le tableau suivant contenu dans le $7^{\mathrm{e}}$ rapport annuel de la commission des services juridiques, tente précisément de répondre à cette question ( $7^{\mathrm{e}}$ rapport annuel, 31 mars 1979, annexe 13, tableau I) :

Couts comparatifs

d'exécution des dossiers d'Aide juridique en 1978 avocats salariés vs pratique privée

\begin{tabular}{lccc}
\hline \multicolumn{1}{c}{$\begin{array}{c}\text { Nature } \\
\text { du } \\
\text { dossier }\end{array}$} & $\begin{array}{c}\text { Coût } \\
\text { avocats salariés }\end{array}$ & $\begin{array}{c}\text { Variation } \\
\text { Coût } \\
\text { pratique privée }\end{array}$ & $\begin{array}{c}\text { pratique privée } \\
\text { P/R } \\
\text { avocats salariés }\end{array}$ \\
\hline $\begin{array}{l}\text { Civil (familial) } \\
\text { (divorce en demande et } \\
\text { en défense) } \\
\text { (séparation en demande } \\
\text { et en défense) }\end{array}$ & $227,32 \$$ & $230,15 \$$ & $1,2 \%$ \\
$\begin{array}{l}\text { Civil (familial) } \\
\text { (autres) }\end{array}$ & & & \\
$\begin{array}{l}\text { Civil (autre) } \\
\text { (assurance-chômage et } \\
\text { pension de vieillesse) }\end{array}$ & 99,61 & 94,04 & $(5,6 \%$ ) \\
$\begin{array}{l}\text { Civil (autre) } \\
\text { (comptes en défense) }\end{array}$ & 38,43 & 77,50 & $101.7 \%$ \\
$\begin{array}{l}\text { Civil (autre) } \\
\text { (autres) }\end{array}$ & 58,28 & 205,33 & $252,3 \%$ \\
\begin{tabular}{l} 
Criminel (total) \\
\hline
\end{tabular} & 62,83 & 168,91 & $168,8 \%$ \\
\hline
\end{tabular}

En dépit de cet effort de clarification, la question faisait de nouveau surface le 15 juin 1981 dans le cadre de l'étude des crédits de l'aide juridique en commission parlementaire. 
M. John Kehoe (député de Chapleau) : C'est justement ma question : Est-ce que cela coûte plus cher pour avoir des permanents? Dans l'ensemble, un mandat qui est confié, soit à des permanents ou à des privés, est-ce que le prix est le même?

M. M.-André Bédard (ministre de la Justice) : Il y a justement une évaluation qui a été faite là-dessus.

Il y a deux ans, une firme comptable qu'on avait engagée pour faire une étude des coûts comparatifs entre ceux qui sont engendrés par le réseau privé et ceux qui sont engendrés par des avocats salariés, nous permettait de dire que, dans le domaine matrimonial, par exemple, c'était le même prix, que ce soit fait soit par un permanent ou par un avocat de pratique privée.

Dans le domaine criminel, cela coûtait deux fois meilleur marché chez nous. Et dans les autres causes civiles, c'était deux fois et demi meilleur marché quand c'était fait par un avocat salarié.

On le voit, l'étude citée n'a ni empêché la question de rebondir, avec tout ce qu'elle sous-entendait de réticences mal dissimulées, ni même nuancer certaines des oppositions traditionnelles. On imagine dès lors à quelle distance nous sommes encore d'une juste perception des diverses données. D'une part, la commission parlementaire n'aura permis à personne de souligner que l'avocat permanent et salarié coûte notablement moins cher que son collègue dans tous les secteurs du nouveau droit social (assurance-chômage, pension de vieillesse, comptes en défense) et dans le domaine du droit criminel. Personne non plus n'aura pointé du doigt que l'entreprise privée ne tient ici son bout que dans le secteur relié de près ou de loin aux questions familiales. D'autre part, personne n'aura pris pleinement conscience d'une faille de taille dans ces calculs : les avocats salariés de l'aide juridique consacrent, en effet, sans qu'on puisse en chiffrer la valeur, des centaines et des milliers d'heures à la création et au maintien de véritables lignes de défense sociale (depuis les caisses populaires jusqu'aux associations contre la violence). Si l'on parvenait à isoler ces coûts, nul doute que la comparaison du coût par dossier serait encore plus favorable à la formule de l'avocat salarié.

Du grief économique, rien, donc, ne subsiste.

\section{La bureaucratisation}

L'aide juridique n'est pourtant pas quitte à si bon compte. En effet, le préjugé populaire à l'endroit des services gouvernementaux 
ne porte pas seulement ni même surtout sur le coût : il concerne aussi la lenteur et l'inefficacité qui, croit-on, se logent automatiquement dans l'intervention étatique. Dans le cas de l'aide juridique, de tels reproches continuent à circuler, même si, heureusement, ils ont presque toujours autant de difficulté à rejoindre la cible.

Tous les chiffres disponibles font état, en effet, d'une productivité accrue chez les avocats salariés. Ainsi, le nombre de dossiers augmente plus vite que le personnel. Ainsi, les délais, comme en témoignent les sondages effectués auprès de la clientèle, semblent, dans l'immense majorité des cas, raisonnables. Ainsi, les bénéficiaires estiment, élément important même si des nuances s'imposent, avoir presque toujours eu gain de cause. Ainsi, dans les cas où les bénéficiaires ont eu la possibilité de recourir tour à tour à des avocats salariés et à des avocats de la pratique privée et peuvent donc comparer valablement les deux formules, la satisfaction de la clientèle ne marque pas de fluctuation notable. Dans ce contexte, il est difficile de crier à la bureaucratisation : lenteur et inefficacité ne semblent pas être ici synonymes de gestion gouvernementale.

Il n'est pas dit que cette heureuse tendance puisse se poursuivre longtemps encore : les permanents de l'aide juridique ont jusqu'à maintenant accumulé une rentable expérience; ils vont commencer peu à peu, ce qui est moins fécond, à prendre de l'âge. Le fait demeure qu'ils ne méritent encore guère le reproche d'embourgeoisement.

Le plaidoyer en faveur d'un plus large recours à l'avocat de pratique privée demeure pourtant vibrant. Encore là, la commission parlementaire qui a procédé à l'examen de la Commission des services juridiques peut utilement servir de barème :

M. John Kehoe (député de Chapleau) : Est-ce une politique globale du ministère d'encourager le citoyen d'aller à la permanence de l'aide juridique? Le principe de base, c'est la liberté de choix, mais la manière que c'est organisé et aussi l'opération de l'aide juridique actuellement fait en sorte que l'aide juridique est dirigée vers l'aide juridique permanente, des avocats qui travaillent pour l'État autrement dit.

M. M.-André Bédard (ministre de la Justice) : C'est définitivement un libre choix. Si telle était l'intention de la publicité que vous évoquez, elle n'a pas grand effet : au contraire, la pratique privée augmente.

M. John Kehoe (député de Chapleau) : Ce n'est pas un libre 
choix à ce moment-là, quand vous êtes obligé d'aller au bureau. 70\%-30\% par exemple, cela fait neuf ans que c'est en opération et c'est encore plus que deux pour un. $22 \%$ à $24 \%$ alors, $6 \%$ à $9 \%$ en neuf ans, et j'ai l'impression avec la publicité qui se fait actuellement, l'obligation du contribuable de se rendre au bureau des permanents n'augmentera pas davantage.

M. J.-C. Rivest (député de Jean-Talon) : C'est quoi la politique?

M. John Kehoe (député de Chapleau) : C'est ce que je vous demande, vous n'avez pas encore répondu. Quelle est l'orientation de la politique de base? Est-ce que vous voulez confier à longue échéance tout aux permanents de l'aide juridique ou avez-vous le libre choix? Ce sont des mots tirés en l'air ?

Un instant plus tard, dans le même cadre, le député Herbert Marx, dont la contribution au droit social est pourtant appréciable, croira donc résumer correctement cet échange en disant :

Je pense que le système fonctionne plus ou moins bien en ce qui concerne les bureaux d'aide juridique et, en ce qui concerne le libre choix, il y a des accrocs comme mes collègues ont soulevé.

Pourtant, le débat en plus de ne révéler aucun accroc du genre, ne contient pas le moindre argument quant à une quelconque supériorité du service offert par les avocats de pratique privée. Tout se déroule donc comme si le présupposé idéologique se servait à luimême de justification suffisante. Si bureaucratisation il y a, les adversaires et les sceptiques se sont soigneusement abstenus de la démontrer. La clientèle, quant à elle, ne retient pas le reproche.

\section{Une passivité entretenue}

Un troisième grief consiste à accuser l'aide juridique d'entretenir et même d'accroître la passivité des bénéficiaires. Articulé, explicite et massif, ce reproche apparaîtra, assez étonnamment d'ailleurs, sous la plume du pédagogue social Jacques Grand'Maison ou plutôt à l'occasion d'un entretien de celui-ci avec un des premiers permanents de l'aide juridique, Me Denis Charette. De larges extraits de l'entrevue méritent de s'insérer ici.

Me Denis Charette: Contrairement aux attentes et aux prévisions, lors de la création du système d'aide juridique, la forme des services dispensés par les avocats de l'aide juridique ne diffère pas sensiblement de celle de pratique 
privée. En effet, les avocats permanents, dans les affaires criminelles et matrimoniales, traitent des dossiers de façon individuelle et exclusivement juridique, de la même façon que les avocats de pratique privée. Ils dispensent donc un service de nature purement curative sans qu'on ait vraiment amorcé tant collectivement pour une clientèle, qu'individuellement pour chacun des clients, des processus de prévention,

(...)

... sans qu'on puisse l'affirmer d'une façon certaine, il m'apparaît que le délinquant qui a à son service, gratuitement, un avocat qui le représente, soit devant le tribunal de la jeunesse, soit devant les différentes cours criminelles pour adultes, se sent plus sécurisé et peut ainsi être tenté de récidiver. Maintenant qu'il a son avocat pour lui obtenir une sentence suspendue, une libération conditionnelle et même le faire acquitter, il y a moins de risque à récidiver.

(...)

La gratuité des services juridiques peut encourager l'irresponsabilité chez la clientèle. En effet, l'on constate que le client d'aide juridique peut se permettre le risque de plaider une cause puisque le fait qu'il perde ou qu'il gagne, a plus ou moins d'incidence réelle chez lui vu qu'il est insolvable. L'insolvabilité d'une partie des clients de l'aide juridique leur permet de prendre des mesures dilatoires, comme de contester des actions sur compte, de contester des demandes d'expulsion d'un logement, d'essayer d'avoir l'autre partie à l'usure dans les causes matrimoniales, etc. Nous n'avons pas réussi à surmonter la dépendance. Nous l'avons même accrue, et c'est ce qui m'inquiète.

(...)

Il est quand même aberrant que les défavorisés, qui obtiennent un service gratuit de l'Etat, doivent se faire représenter par des avocats fonctionnaires pour faire valoir leurs prétentions devant des fonctionnaires qui font mal leur travail. Si l'on pousse cette logique jusqu'au bout, faudra-t-il un jour, lorsque le système d'aide juridique sera complètement fonctionnarisé, lorsqu'il faudra deux mois pour avoir un rendez-vous, lorsque les services seront inadéquats, que les défavorisés puissent avoir recours à d'autres formes d'aide juridique? Certes, cette forme d'intervention est peut-être nécessaire à court terme, mais ce n'est certes pas de la prévention.

(...)

... force est de constater que très souvent l'information est mal reçue; la clientèle l'interprète mal; et il eût mieux valu que la personne n'ait pas du tout d'information 
plutôt que de l'interpréter de cette manière. Souvent, il faut que cette information soit appuyée d'une entrevue avec un avocat, et à ce moment-là, nous ne sommes pas plus avancés.

(Grand'Maison, Jacques, De quel droit ?, tome 2, La pratique sociale, Leméac, 1980, page 136 et suivantes).

De tels propos, qui ont sans doute le mérite de souligner les limites de l'intervention strictement curative, sont, d'autre part, si lourds de sophismes et si gros de préjugés ténébreux qu'il faut y lire bien plus qu'une simple évaluation d'un service public. S'y déploie, en effet, en termes candidement rétrogrades, une complète remise en question de nos plus indiscutables acquis sociaux.

Bornons-nous à des exemples obvis :

- défendre gratuitement des défavorisés, est-ce les encourager à multiplier les actes de délinquance ?

- est-il aberrant de fournir au citoyen de quoi faire face aux interventions de l'Etat?

- l'information est-elle, ainsi que l'affirmait un ancien premier ministre québécois à propos de l'instruction, "une chose que certains portent aussi mal que la boisson " ?

- les insolvables sont-ils, plus que les autres, enclins à abuser des services gratuits qu'on leur offre?

Autant de questions, on l'admettra, qui ne visent pas la gestion présente du service d'aide juridique québécois, mais l'ensemble des rapports entre l'État et le citoyen.

En un sens, l'ampleur même de la remise en question dispense d'une réplique point par point. L'aide juridique en effet, n'est responsable à elle seule ni de la grandeur ni de la décadence de l'Occident...

Un élément, pourtant, dans les propos tenus par monsieur Charette, se circonscrit suffisamment pour permettre un examen valable : il a trait à l'accent que mettrait l'aide juridique québécoise sur la seule dimension curative au détriment d'une prévention pourtant infiniment plus salvatrice.

Sur ce terrain, la critique frappe dans la bonne direction, mais beaucoup trop fort. D'une part, on caricature injustement l'aide juridique si l'on réduit tous ses efforts à une sorte de "pathologie judiciaire ". D'autre part, si l'on prétendait attendre de l'aide juridique la transformation radicale de la société et même la parousie, il faudrait néanmoins que les avocats qui rendent ce service s'attaquent de façon directe et immédiatement efficace aux maux qui as- 
saillent les démunis d'aujourd'hui. La révolution, le Grand Virage, la Totale Illumination, tout cela est sans doute fort bien, mais des dossiers sont là qui charrient la chair et le sang. Ils réclament non pas un éventuel réalignement des valeurs, mais un correctif modestement disponible aujourd'hui même. Et cela aussi est respectable, authentique, indispensable.

Certes, dans les faits, l'aide juridique ne doit jamais perdre de vue qu'il y a quelque chose de vicié dans les règles du jeu elles-mêmes et qu'il faut se battre pour des règles moins injustes. Mais elle doit pourtant, au nom du droit de l'individu à ne pas toujours attendre le jugement dernier et la lointaine réhabilitation de l'Espèce, jouer le jeu.

L'équilibre est-il atteint ? Peut-être pas. Il n'est pourtant pas si loin. L'aide juridique a fort bien su, en effet, dans tel et tel cas, au lieu de défendre un à un les centaines de laissés pour compte, orchestrer contre l'État lui-même l'assaut qui a conduit au changement législatif ou à une moindre arrogance réglementaire. Qu'on songe ici aux trop-perçus que l'État a voulu subitement réclamer des assistés sociaux. Elle a su aussi, face à maints projets de loi, intervenir au bon moment, c'est-à-dire au moment même où les élus s'apprêtaient à modifier les règles du jeu. Tout cela dépasse très largement ce qu'il est convenu d'appeler le curatif.

Quant au travail de prévention qui viserait à regaillardir non pas tant la structure et la loi, mais le citoyen lui-même, comment, à la lecture des rapports annuels de l'aide juridique, prétendre que l'aide juridique y ait renoncé ou même qu'elle le néglige ? Certes, on trouve ça et là, dans les textes qui émanent des divers bureaux régionaux, des pages larmoyantes ou exaspérées où des permanents surchargés se disent incapables de soigner à la fois la société et les individus. Malgré tout, ce ton, émotif et facilement hargneux, témoigne des fatigues individuelles beaucoup plus qu'il ne révèle de la part du réseau d'aide juridique l'intention ferme de se concentrer sur le seul curatif.

À côté de ces quelques paragraphes hérissés, on trouve d'ailleurs dans les documents de l'aide juridique la trace de dizaines de contributions intelligentes et généreuses à un vaste travail de prévention et à la construction au niveau du vécu de solides lignes de défense.

Quant à savoir si l'aide juridique, comme le voulait notre citation, encourage les pauvres à abuser ou leur communique une information que leurs pauvres cerveaux ne peuvent absorber, il serait 
inutile de s'embarquer dans semblable débat. Droite et gauche, en effet, n'en sont plus à leurs premiers affrontements et rien ne permet de croire que l'une des deux va prochainement admettre ses torts et déclarer forfait. Mieux vaut donc que l'aide juridique se résigne à vivre longtemps encore en face de gens qui préféreraient garder les avantages de l'État pour les «bien-méritants »...

Des trois griefs couramment évoqués, aucun ne ternit vraiment un bilan globalement positif.

\section{LES PROCHAINES ÉTAPES}

Bilan positif, donc, mais aussi simple étape. Sur chacun des fronts familiers, l'aide juridique doit, en effet, prévoir de nouveaux assauts et inventer de nouvelles mesures de rétorsion. Les problèmes de coût, d'embourgeoisement et d'atomisation ne sont ni ne seront jamais complètement résorbés. Ils en sont tout simplement à de nouvelles incarnations.

\section{Le principe du libre choix}

Controversé au départ, le principe du libre choix l'est tout autant aujourd'hui. Heureusement, les années écoulées permettent de porter sur ses conséquences exactes un jugement plus ferme encore qu'en 1972.

Ce qu'on soupçonnait s'est en effet vérifié : il en coûte autant et plus - et même beaucoup plus - pour le dossier confié à la pratique privée que pour le dossier traité par un avocat salarié. Ce que révélait déjà la pratique étrangère s'est également confirmé : l'immense majorité des démunis ne trouvent aucun avantage concret à se prévaloir de cette fameuse liberté de choix puisqu'ils ne connaissent littéralement aucun avocat. Enfin, ce qu'on pouvait redouter est aussi en train de se produire : en période de compression budgétaire et de moindre embauche, on optera paradoxalement pour l'échappatoire la plus coûteuse, c'est-à-dire le recours plus massif aux avocats de pratique privée. En d'autres termes, le libre choix des bénéficiaires, comme par hasard, se portera plus souvent vers la pratique privée lorsque les avocats salariés seront particulièrement surchargés. D'où un lien étroit entre la dimension économique et le principe du libre choix.

Traduite en termes concrets, cette évolution accule l'aide juridique à un choix brutal : maintiendra-t-on ou non le principe du libre choix? Si on s'y cramponne, on peut parier que les coûts croissants et incontrôlables (par définition) de ce secteur de l'aide juridique vont conduire l'ensemble du programme à la camisole de force. 
Au niveau de la Commission des services juridiques, on répugne visiblement à évoquer la possibilité d'une telle modification. On souligne, avec ce qui ressemble à une conviction sincère, que la concurrence a toujours des avantages et on se félicite d'avoir ainsi une sorte de secteur-témoin qui permet de désarmer la critique.

Cette position, relativement confortable, est devenue fragile. Peut-être même est-elle suicidaire. Ou bien l'aide juridique entreprend la bataille qui la conduira au contrôle de tous ses coûts, même s'il lui faut remettre en question le principe du libre choix, ou bien elle laisse grimper de façon affolante et incontrôlée le coût des mandats donnés à l'extérieur. Dans ce cas, elle recevra tôt ou tard un superbe coup de rabot de la part du Conseil du trésor.

\section{Le danger d'embourgeoisement}

Comme tous les services qui vieillissent dans un relatif succès, l'aide juridique fait face à la menace de l'embourgeoisement. Elle y a, certes, assez bien résisté jusqu'à maintenant et strictement rien, avons-nous constaté, ne permet encore de la croire empoussiérée. L'avenir, toutefois, se présente autrement.

Ici encore, en effet, il faut prendre conscience de la conjoncture économique et sociale. De pesante façon d'ailleurs, cette conjoncture limite le nombre des emplois disponibles, réduit la mobilité des moins doués, garde chacun dans l'emploi qu'il occupe. Alors qu'une situation économique moins étouffante accentuerait un certain nomadisme et une relative audace, les présentes insécurités, dans le monde du droit comme ailleurs, incitent plutôt à la stabilité et au conservatisme.

À cela s'ajoute le fait que l'aide juridique, qui constituait une nouveauté et un défi il y a huit ou neuf ans, fait désormais partie des empires stables. La tentation est donc forte aujourd'hui d'y chercher refuge ou d'y rester peinard. Le dire, ce n'est que constater l'inévitable : ceux qui persistent sur leur paquebot ne ressemblent pas nécessairement à ceux qui s'aventuraient sur le canot d'écorce.

Le problème n'est pas nouveau? Oui, il l'est. Il l'est par le contexte et par les hommes: d'une part, l'heure semble exiger de l'aide juridique plus de gestion que d'inventivité; d'autre part, l'expérience qui a augmenté la productivité des permanents de l'aide juridique donne maintenant un air de routine fastidieuse à un nombre croissant de dossiers.

Bel exemple d'une machine condamnée à la fuite en avant, l'aide juridique doit, sous peine d'une rapide sclérose, approfondir 
et déborder ses tâches classiques. Ce qui ne signifie surtout pas que l'acquis mérite la liquidation.

\section{Entre l'atomisation et l'utopie}

Autre conséquence de la conjoncture, les gouvernements de tout acabit sont aujourd'hui tentés de sabrer dans leurs responsabilités et surtout dans leurs dépenses sociales. Depuis l'éducation jusqu'à l'assurance-chômage, depuis la santé jusqu'à l'aide juridique, tous et chacun des programmes orientés vers le soutien des démunis et la réduction des disparités s'en trouvent menacés. Or, on en sait aujourd'hui assez long sur les corollaires de la pauvreté et du dénuement pour prévoir dès maintenant les retombées de cette nouvelle campagne d'austérité : les pauvres en feront les frais.

Les faiblesses, en effet, ont ceci de particulier qu'elles s'agglutinent, s'accumulent, se confrontent les unes les autres. Le pauvre meurt plus jeune, l'handicapé jouit d'un moindre salaire, la famille monoparentale déménage plus souvent, etc.

Dès lors, comment peut réagir un service d'aide juridique : au niveau du remède ou au niveau du cataplasme ? Là non plus, en effet, on ne peut invoquer les succès obtenus dans le passé. Certes, on a assez bien réussi hier à maintenir l'équilibre entre l'indispensable intervention en faveur des individus et la pression sur les macro-décisions, entre l'atomisation du geste et l'ampleur de la conception, mais ceci ne garantit pas grand chose. Un nouvel équilibre doit s'inventer, qui amplifiera sans doute la dimension collective.

L'aide juridique québécoise, somme toute, est en bonne santé. Mais être en bonne santé quand on monte au front, est-ce une bien rassurante garantie?

\section{BIBLIOGRAPHIE}

Articles de L. Laplante.

GRAND'MAISON, Jacques (1980): De quel droit ?, tome 2 : La pratique sociale, Montréal, Leméac.

(QUEBEC) ASSEMBLEE NATIONALE (1980) : Journal des débats, Commissions Parlementaires, $4^{e}$ Session, $31^{e}$ Législature. Commission permanente des Affaires intergouvernementales. Etude des crédits du ministère des Affaires sociales, le 9 juin - no 297 (Président : M. Clément Richard).

(QUEBEC) COMMISSION DES SERVICES JURIDIQUES (1979) : Droit de la famille, droit des femmes ? En collaboration avec le Conseil du statut de la femme. Dossier d'information à l'intention des femmes. Québec, Éditeur officiel. 